\title{
Mille ans de peu d'importance? Les éthno-préfixes « gallo »- et « franco »- et le message de leur usage : mono- versus polycausalité
}

\author{
Frank JODL \\ Universität Siegen \\ jodl@romanistik.uni-siegen.de
}

\begin{abstract}
RESUME
Pour trouver des explications convaincantes quant à des changements linguistiques ayant eu lieu dans des époques qui nous empêchent l'accès direct on ne peut pas se passer ni d'un seul facteur potentiel. Il s'est même avéré que ce sont les explications polycausales qui sont les plus prometteuses. Mais force est de constater que dans bien des cas cette règle n'a pas été respectée. La surestimation du rôle joué par le substrat et par conséquent du rôle de l'antiquité pour expliquer la naissance du français transparaît dans le fait que l'ethno-préfixe " gallo- » s'est établi comme terme consacré, ce qui n'est pas le cas pour son corrélatif «franco-». Mais ce même phénomène concerne aussi d'autres problèmes de glottogénèse comme celui de la naissance du protoromanche.
\end{abstract}

Mots-clé: naissance du français, "gallo-", "franco-", linguistique romane.

[Recibido, enero 2012; aprobado, junio 2012]

\section{A thousand unimportant years? The ethnical preffixes "gallo" and "franco" and the message behind their use: mono or polycausality?}

\begin{abstract}
To find convincing explanations for linguistic changes that have occurred in periods that prevent us direct access can not do without either one potential factor. It has even been proved that it is polycausal explanations that are the most promising. But it is clear that in many cases this rule has not been followed. The overestimation of the role played by the substrate and therefore the role of antiquity to explain the birth of French reflected in the fact that ethno-prefix "Gallo" has established itself as dedicated term, which is not the case for its correlative "Franco-." The same phenomenon also applies to other linguistic problems such as the birth of protoromansch.
\end{abstract}

Keywords: origins of French, gallo, franco, Romance linguistics. 


\section{Introduction}

Après la superstratomanie ${ }^{1}$ des années trente du XXè siècle et le déclin de l'intérêt pour le rôle des contacts langagiers du côté des linguistes qui a ensuite marqué la discipline pendant les années cinquante et soixante, on peut constater qu'à partir des années soixante-dix l'influence qu'ont pu exercer les contacts linguistiques par rapport au changement langagier et à la naissance de nouvelles langues $^{2}$ a été revalorisée, cf. à propos du rôle du superstrat franc Schmitt (1974: $61 \mathrm{~s}$.$) :$

L'apport du superstrat germanique [...] a certainement été important, personne ne le contestera. La vue de v. Wartburg concernant la symbiose du francique et du galloroman du Nord est acceptable dans l'ensemble. Les conclusions qu'il en a tirées sont cependant trop générales et absolues [...]. Ce ne sont pas les Francs qui ont créé le protoroman de couleur française, ni les Burgondes, le protofrancoprovençal. Ces unités existaient à leur arrivée et ils n'ont pu que modifier un moule préexistant [...]. Elle [la Galloromania] apparaît ainsi, en fin de compte, comme le résultat d'un amalgame de facteurs historiques, politiques, ethniques, culturels et naturels les plus divers.

Cerquiglini ( $\left.{ }^{3} 2007: 53\right)$, lui aussi, attribue un poids immense au Moyen-Age et à la situation de contact linguistique correspondant («superstrat») qu'il considère comme facteur décisif pour la naissance du (proto-)français :

L'influence germanique, tout d'abord, eut un double effet : Elle a distingué le protofrançais des autres langues romanes (à commencer par la protolangue d'oc, au sud de la Loire), [...] elle lui a donné une coloration définitive, ce qui peut avoir hâté la prise de conscience de son altérité face au latin.

Kramer (1999: 19) prétend appliquer cette manière de voir les choses à TOUTES les langues romanes, cf. Id. ibd. :

Anders als Robert de Dardel und Jakob Wüest (1993) es sehen, fällt die Geburtsstunde der romanischen Sprachen nicht mit der Expansion des imperium Romanum zusammen, sondern vielmehr mit den Wirren nach dessen Untergang. Die Geschichte des Italienischen beginnt also nicht mit der Unterwerfung der Italiker 265 v. Chr., die Geschichte des Spanischen nicht mit der Einrichtung der Provinz Hispania 197 v. Chr. und die Geschichte des Französischen nicht mit Caesars Sieg 50 v. Chr., sondern irgendwann zwischen 600 und 800 n. Chr. zerreißen die nicht mehr durch einheitsfördernde Instanzen gebändigten

\footnotetext{
${ }^{1}$ Pour un résumé de la situation cf. JodL (2004: 8-11) ou SCHLEMmer (1983).

2 Malgré LÜDTKE $(2005: 54)$ nous parlons ici de la «naissance de nouvelles LANGUES », cf. JODL (2008).
} 
Zentrifugalkräfte das gesprochene Latein, und die Entwicklung der romanischen Idiome nimmt ihren Lauf. ${ }^{3}$

Cette revalorisation du facteur du superstrat tient aussi compte d'un principe méthodologique dont la portée ne peut point être sousestimée : en l'occurrence celui de considérer la naissance du protofrançais comme un phénomène qu'il faut examiner sous l'aspect de la polycausalité. Schmitt (1974: 61s., cité plus haut) y a déjà fait allusion dès 1974 en décrivant [les idiomes de] la Galloromania comme « résultat d'un amalgame de facteurs historiques, politiques, ethniques, culturels et naturels les plus divers. »

Dans ce contexte, il faut aussi mettre en relief qu'aucune des explications monocausales ${ }^{4}$ proférées pour décrire la naissance d'une langue ne s'est jamais avérée convaincante : Il faut non seulement prendre en compte tous les facteurs extralinguistiques sans priviligier une certaine époque historique au détriment d'une autre, mais aussi se demander quel rôle pouvaient jouer les facteurs internes au système de la langue $\mathrm{e}^{5}$ au sein de 1'amalgame de facteurs. En ce qui concerne le rapport existant entre facteurs externes et internes ${ }^{6}$ au système de la langue - qui représente un aspect tout particulier de la polycausalité - nous renvoyons à la description qu'en donne Argente (1998:9) : «Factores estructurales [= facteurs internes au système de la langue, F.J .], en suma, determinarán tal vez cuáles sean los cambios posibles y los cambios previsibles, pero poco aportarán acerca del proceso histórico concreto que haya conducido a ellos. »

Il est curieux de constater que cette orientation vers le principe de la polycausalité n'est pas toujours respectée, qu'il s'agisse de travaux récents ou plus anciens. Nous tenons à signaler que ce n'est pas le cas en ce qui concerne l'opinion de Kramer (1999 : 19, cité plus haut) : Au premier abord, on serait tenté de voir cette explication comme une tentative de survalorisation du facteur du superstrat, ce qui aurait comme conséquence de tomber dans le piège des explications monocausales. Ce qu'on peut y voir c'est - à notre avis - plutôt l'objectif d'identifier parmi la variété des facteurs celui qui a accéléré l'évolution vers la naissance des langues romanes et sans lequel les autres facteurs, soit internes soit externes au latin, n'auraient pas pu se répercuter sur l'évolution de la langue en question. Pour dé-

\footnotetext{
${ }^{3}$ « Contrairement à ce qu'affirment Robert de Dardel et Jakob Wüest (1993), la naissance des langues romanes ne coïncide pas avec l'expansion de l'empire romain mais plutôt avec les vicissitudes qui entraînèrent sa chute. L'histoire de l'italien ne commence donc pas par l'assujettissement des peuples italiques, advenu en 265 av. J.-C., l'histoire de l'espagnol ne commence pas par l'établissement de la province de l'Hispania en $197 \mathrm{av}$. J.-C. et l'histoire du français ne commence pas par la victoire de César en 50 av. J.-C. mais entre 600 et 800 après J.-C. quand les forces centrifugales, qui ne sont plus apprivoisées par les instances encourageant l'unité, déchirent le latin parlé et l'évolution des idiomes romans prend son envol. »

${ }^{4}$ Comme p.ex. celle de WARTBurg $\left({ }^{5} 1946: 65\right)$ proférée à propos de la diphtongaison qui a été atténuée entre autres par HiLTY (1992 : 120s.).

${ }^{5}$ Nous avons essayé de trouver un mode de description polycausale dans JODL (2004, surtout 97-115 et 309-312), cf. aussi JoDL (2008: 165-175).

${ }^{6}$ Dans la citation à suivre, les facteurs internes à la langue sont nommés « factores estructurales ».
} 
montrer les dangers des explications monocausales il existe d'autres cas. Nous voudrions en donner deux exemples actuels dont l'un concerne la naissance du (proto-)romanche et l'autre celle du (proto-)français.

En ce qui concerne le (proto-)romanche, il se manifeste une forte tendance à ancrer les évolutions langagières les plus significatives dans l'antiquité et de contester à l'époque médiévale la capacité d'avoir pu amener des changements linguistiques majeurs. Dans son travail «Wann wurde Graubünden wirklich romanisiert? (Quand les Grisons furent-ils vraiment romanisés) », Hilty (2008: 224) arrive à la conclusion suivante:

Ich bin überzeugt, dass die Besonderheiten des Bündner Lateins in jener Sprache vorhanden waren, die [...] den Raum der Raetia Prima um 350 [n. Chr.] vom Bodensee bis zur Alpenwasserscheide ausfüllte. Um diese Zeit war das ProtoBündnerromanische konsolidiert. Eine Erweiterung und Verstärkung erfolgte anschliessend noch durch die Kirchensprache. Flüchtlinge aus dem Norden hatten sich wohl anzupassen, sofern ihre Sprache abwich. Wann wurde Graubünden wirklich romanisiert? Zwischen 15 v. Chr. und der Mitte des 4. Jahrhunderts wurde die Raetia Prima [...] so romanisiert, dass in diesem Raum das Bündnerromanische als selbständige romanische Sprache entstehen konnte (cf. Hilty 2007). ${ }^{7}$

Le message principal de ce travail est l'affirmation « qu'à cette époque [= vers 350 apr. J.-C.], le proto-romanche s'était déjà consolidé. » Une telle interprétation des faits historiques comporte pourtant le risque, nous l'avons dit plus haut, de contester à toute une époque, marquée d'ailleurs par les constellations extralinguistiques les plus décisives, la capacité d'avoir contribué à forger le caractère de la nouvelle langue.

Si les recherches des historiens avait abouti au résultat que les Grisons n'ont pas été soumis à partir de l'an 500 env. à des conditions extralinguistiques capables, selon les mécanismes universels des contacts linguistiques, de forger le protoromanche à partir du latin parlé dans les Grisons, on comprendrait pourquoi il vaudrait mieux se concentrer sur l'antiquité pour trouver les facteurs qui ont donné naissance à cette nouvelle langue. Mais celà n'a pas été le cas, bien au contraire, cf. Hilty (2008: 220s) :

Felix [sic] Walser ist nicht der einzige Forscher, der für eine späte Romanisierung plädiert. Seine Auffassung ist nur die extremste. Er und die

\footnotetext{
${ }^{7}$ « Je suis convaincu que les particularités du Latin des Grisons étaient présentes dans la langue qui vers 350 [apr. J.-C.] - remplissait l'espace de la Raetia Prima du Lac de Constance jusqu'à la ligne alpine de partage des eaux. A cette époque, le proto-romanche s'était déjà consolidé. Il s'ensuivit plus tard une amplification et un renforcement sous l'influence du langage écclésiastique. On peut supposer que les réfugiés venant du Nord durent s'adapter linguistiquement dans le cas où leur langue divergeait de celle parlée dans les Grisons. Quand les Grisons furent-ils vraiment romanisés? Entre l'an 15 av. J.-C. et le milieu du 4è siècle, la Raetia Prima fut romanisée au point de permettre l'émergence, dans cet espace, du Romanche comme langue romane indépendante. »
} 
anderen Befürworter einer späten Romanisierung müssen aber nicht nur erklären können, wann die Romanisierung stattgefunden hat, sondern vor allem auch wie sie stattgefunden hat. ${ }^{8}$

Mais, comme le précise aussi M. Hilty, ce n'est pas le critère du «quand » de la latinisation ${ }^{9}$ qui fournira la clé pour pourvoir décrire les circonstances de la naissance du protoromanche : que le latin ait joué un rôle important dans notre zone dès l'époque impériale ne sera contesté par personne. Si pourtant on se pose la question de savoir quels étaient les facteurs qui ont transformé ce latin d'une manière si particulière, il ne suffira point de répondre à la question du « quand » de la latinisation. Il faut aussi prendre en considération les résultats des spécialistes d'archéologie et d'histoire médiévale qui donnent une autre image de la situation, cf. pour un résumé Jodl (2004 : 153-206), et qui nous permettent d'aborder le problème du « comment». En d'autres termes : «Pourquoi devrait-on exclure délibérement toute une époque, en l'occurrence le Moyen Age, et avec elle tout un faisceau de facteurs qui pourraient éclairer des problèmes linguistiques ? "

Par conséquent, il n'est pas nécessaire de reprendre la discussion sur le « quand» de la latinisation des Grisons - si elle s'est achevée vers 350 apr. J.-C. ou plutôt vers 500 apr. J.-C. - la question cruciale est de savoir s'il est possible d'expliquer l'évolution de notre "strate", qui est le latin des Grisons, en omettant purement et simplement l'une des strates qui forment, toutes ensemble, le mécanisme qui amorce et régit, en combinaison avec les facteurs internes, le changement linguistique, cf. Schmitt (1974: 61s.), cité plus haut, et Jodl (2008: 165-174) ou Argente (1998: 9, cité plus haut).

Abordons le problème sous un angle différent : Qu'est-ce qui transforme le latin parlé des Grisons en protoromanche, quels en sont les traits langagiers concrets ? Ce sont, pour en nommer les traits les plus significatifs, la diphtongaison spontanée $\mathrm{de} / \mathrm{e} / \mathrm{et} / \mathrm{o} /$ fermés en syllabe ouverte ; la palatalisaton de $[\mathrm{k}, \mathrm{g} / \mathrm{a}]$ et la chute $\mathrm{du}-\mathrm{O}$ final.

Partir du principe que toutes ces évolutions cruciales se sont déjà produites jusque vers 350 apr. J.-C. signifierait que le Moyen Age, une période marquée par des constellations extralinguistiques extraordinaires de plus de 1000 ans, se serait limité à contribuer à la naissance du protoromanche des évolutions secondaires comme des emprunts et des calques lexicaux voire syntactiques.

Que la portée des effets extralinguistiques se soit limitée de cette manière ne semble pas très probable si on considère Kramer (1999: 19) et Schmidt (1974 : 61s.) cf. citations plus haut: Pourquoi un mécanisme voire une époque dont on a

\footnotetext{
${ }^{8}$ «Felix Walser n'est pas le seul chercheur à plaider pour une romanisation tardive. Mais ce qui le distingue des autres, c'est sa position extrème. Pour être convaincants, lui et les autres défenseurs d'une romanisation tardive doivent toutefois expliquer non seulement quand a eu lieu la romanisation mais aussi comment elle s'est réalisée. »

${ }^{9}$ Nous suivons ici la proposition qu'a faite BANNIARD $(1996: 577)$ de faire la distinction entre "latinisation" et "romanisation".
} 
fait valoir l'importance pour l'évolution du (proto-)français, cf. Schmidt (1974 : 61s.), ou des langues romanes en général, cf. Kramer (1999: 19), devrait-il être écarté du spectrum des facteurs qui ont pu influencer l'évolution du latin parlé dans les Grisons? Nous y reviendrons plus bas. Reste à signaler que la reconstitution des conditions extralinguistiques qui s'étaient développées dans les Grisons à l'époque protomédiévale et de l'importance du superstrat francoroman et franc dans cette zone présentée dans Jodl (2004 : 153-206) et Jodl (2005) devrait inciter à ne pas sousestimer la vigueur de cette influence. Il existe des indices, cf. Jodl (ibd.), qui laissent supposer à propos de la vigueur du superstrat rencontrée dans les Grisons après l'arrivée d'individus francoromans (ou carrément francs) était suffisamment proche à celle rencontrée en Gaule septentrionale après l'arrivée des francs pour avoir des conséquences langagières du même ordre.

Passons à l'exemple concernant le français que nous avions annoncé plus haut. Bien que le rôle décisif du superstrat franc dont nous avons traité ne soit plus contesté, il faut signaler ici que cet état de fait ne se reflète pas dans la terminologie spécifique de la discipline : le terme de « francoroman » est très peu ou presque pas employé en linguistique romane. Élément de la théorie des strates, lui aussi, il n'a pas eu le même succès que son corrélatif, le concept de "galloroman » qui représente le résultat du substrat. Nous en trouvons une première preuve dans le fait que le terme de « francoroman » / « franco-roman » ne figure pas dans les dictionnaires français.

Il est également difficile de le trouver sur Internet ${ }^{10}$. Il n'y a que 15 occurrences intéressantes de «franco-roman » voire «francoroman», tandis que le terme de « gallo-roman » comptabilise 116.000 occurrences.

Si nous recherchons sur Google les termes en question en d'autres langues importantes pour la linguistique romane, nous trouvons :

En italien : 2 occurrences pour "francoromanzo"contre 2630 occurrences pour "galloromanzo"

En allemand : 138 occurrences pour "frankoromanisch" contre 10600 occurrences pour "galloromanisch"; 1 occurrence pour "francoromanisch"

En anglais : 26 occurrences pour "francoromance" contre 10800 occurrences pour "galloromance"

En espagnol: 0 occurrences pour "francorromance" contre 847 occurrences pour "galorromance"

Il existe, il est vrai, un "Frankoromanistenverband" (Association des francoromanistes allemands) etc., mais dans les descriptions philologiques ou linguistiques relatives à la naissance du français voire des langues romanes, le terme de "francoroman" est relativement peu usité ${ }^{11}$. Ceci est d'autant plus déplorable que la théorie des strates - dont "francoroman" fait partie en jouant le rôle de "résultat du supers-

\footnotetext{
${ }^{10}$ Il faut bien évidemment exclure les occurrences de «franco-roman » qui en roumain désignent toute autre chose.

${ }^{11}$ Pour des exemples positifs cf. entre autres Gsell (1997: 144ss.).
} 
trat" - est particulièrement bien appropriée ${ }^{12}$ à décrire l'évolution du latin vers le français et aussi à clarifier la position particulière du français par rapport aux autres langues romanes, à condition toutefois de mentionner toutes les strates en jeu, et surtout celles qui ont contribué le plus à la formation des traits typiques de la nouvelle langue. $\mathrm{Vu}$ les circonstances historiques de la naissance du français et les résultats langagiers qui en découlent, il faut même dire, vice-versa, que l'histoire du français se prête particulièrement bien à l'illustration de l'essence de la théorie des strates. Il faudrait en tenir compte dans la terminologie en question pour ne pas donner une fausse idée des faits : Les diagnostics donnés par Schmitt, Cerquiglini et Kramer ne s'accordent pas avec le choix du terme " galloroman » pour désigner scientifiquement, entre autres, le français. Quelle raison se cache derrière la préférence pour «galloroman »? Est-ce lié au fait que certains linguistes insistent sur les racines antiques des langues romanes, comme c'est le cas pour le romanche, cf. plus haut?

\section{La thèse de l'importation du francoroman en Italie du Nord et la discussion actuelle : $L$ 'exemple de la palatalisation de $[\mathrm{k}, \mathrm{g} / \mathrm{a}]=$ PKA}

L'orientation voire la ré-orientation vers l'antiquité comme époque décisive par rapport à la naissance des langues romanes dont nous avons traité, ne concerne non seulement le français tout seul ou bien le romanche tout seul, mais aussi la théorie selon laquelle la naissance du romanche serait due au contact avec le francoroman voire le protofrançais.

Quelle est donc la situation particulière qui s'est établie dans les Grisons au début du Moyen Age et qui a pu avoir des conséquences décisives sur le latin parlé dans cette région?

Dans Jodl (2004 : surtout 128-144) et Jodl (2005), nous avons essayé de retracer - à titre d'exemple pour tout le faisceau d'innovations qui caractérise les deux langues - la possibilité de voir la palatalisation de $[\mathrm{k}, \mathrm{g} / \mathrm{a}]$ (= PKA) advenue en padano-rhétoroman comme un phénomène causé par le contact linguistique avec le francoroman ce qui nous paraissait l'explication la plus probable à propos de l'origine de ce parallélisme.

Étant parti des nouveaux résultats historio-archéologiques qui nous fournissent une autre image de la colonisation franque en Gaule et de la situation de contacts linguistiques entre les locuteurs du gallolatin et ceux de l'ancien francique, nous sommes arrivés à la conclusion que c'était au sein de la noblesse franque, suffisamment bilingue, que sont nés les traits langagiers décisifs, comme la PKA (« fränkisch geprägte Sonderlatinität ${ }^{13} /$ « latinité particulière d'empreinte francique »).

\footnotetext{
${ }^{12}$ Evidemment, cette théorie a été contestée et rejetée à plusieurs reprises pour être en fin des comptes revalorisée ces temps derniers (cf. JODL 2004: 10 pour un sommaire). Cette revalorisation est bien entendu aussi due aux succès qu'ont eus les recherches modernes sur le rôle du contact linguistique tout court.

${ }^{13} \hat{A}$ propos de ce sujet il y a une erreur dans Heinemann (2005:215s.): Selon ce travail, le noyau du travail Jodl (2004), c'est-à-dire la «fränkisch geprägte Sonderlatinität» = «latinité particulière
} 
Une fois établie en France, cette "latinité particulière d'empreinte francique » qui portait en elle tous les traits langagiers qui nous intéressent, comme la PKA, pouvait se répandre jusqu'en Italie septentrionale à travers l'expansion politique des Mérovingiens et surtout des Carolingiens après 774 apr. J.-C.

En ce qui concerne la question de la PKA, on ne peut pourtant pas cacher qu'un nombre important de romanistes est soit sceptique, comme Schmid (1956), soit totalement opposé à la thèse de l'importation de la PKA francoromane en Italie du Nord, comme Pfister (1995 : 196) et Kristol (1998 : 944).

Or, il est intéressant de noter que récemment le thème de l'origine du parallélisme qui existe entre le domaine d'oïl et le domaine padano-rhétoroman au sujet de la PKA a été repris dans le cadre d'une théorie selon laquelle la PKA padanorhétoromane remonterait à une importation de la Francoromania, cf. p. ex. Jodl (2000), (2004) et Jodl $(2005)^{14}$. Ainsi nous trouvons maintenant dans Wüest (2008: 379) :

Trotzdem möchten wir nicht annehmen, dass es sich in Gallien und in Norditalien um zwei getrennte Erscheinungen handelt. Dies mag zwar einleuchten, wenn man die Palatalisierung von C und G vor A isoliert betrachtet. Die gemeinsamen Lautentwicklungen sind aber zu zahlreich, als dass soviel Übereinstimmung[en] zufällig sein können [sic]. ${ }^{15}$

Ce qui frappe par ailleurs, c'est le fait qu'on puisse - même si leurs convictions quant à la valorisation des facteurs décisifs pour la naissance du protoromanche s'opposent diamètralement ${ }^{16}$ - observer un parallélisme d'argumentation entre Hilty (2008) et Wüest (2008). Autrement dit, bien que le but de l'article de Wüest (2008) soit de souligner, en s'appuyant sur une conférence du grand maître József

d'empreinte francique » serait «weitgehend frei [sic!] von fränkischen Merkmalen » = « en grande mesure exempte [sic!] de traits franciques. Mais comment est-ce qu'une latinité particulière « d'empreinte francique » (cf. Jodl 2004: 93) pourrait être « exempte de traits franciques »? Il doit y avoir eu un malentendu.

${ }^{14}$ Les travaux JODL $(2000,2004,2005)$ sont dédiés explicitement à la naissance de la PKA dans les deux zones. Il ne faut pas oublier que Otto Gsell avait déjà abordé ce thème en 1996, cf. GSELL (1996 : 576) : «Umgekehrt kann bzw. könnte eine gesicherte Datierung auch positiv zur Herkunftsfrage eines Wandels beitragen: Falls oberit.-rätorom. [u> y] und $[\mathrm{k} / \mathrm{a}>\mathrm{kj}]$ nach dem 7 . Jh. beginnen, wäre Import aus der Francia wahrscheinlicher als autochthone Parallelentwicklungen beiderseits der Alpen; [...]. » [Trad. F.J.: « Vice-versa, une datation assurée pourrait contribuer de manière positive à éclairer la question sur l'origine d'un changement linguistique : si l'évolution padanorhétoromane $[\mathrm{u}>\mathrm{y}]$ et $[\mathrm{k} / \mathrm{a}>\mathrm{kj}]$ ne commence qu'après le VIIè siècle, une importation de France serait plus probable que deux évolutions autochtones en parallèle des deux côtés des Alpes; [...].]. »

${ }^{15}$ « Malgré tout, nous ne pouvons pas accepter qu'il s'agisse de deux phénomènes distincts, l'un apparu en Gaule et l'autre en Italie du Nord. Ceci pourrait sembler être le cas si on considère la palatalisation de $\mathrm{C}$ et $\mathrm{G}$ devant $\mathrm{A}$ comme cas isolé. Les innovations phonétiques que les deux zones ont en commun sont toutefois trop nombreuses ${ }^{15}$ pour être accidentelles. »

${ }^{16}$ Selon Hilty $(2008: 224)$ : Latinisation et naissance du protoromanche achevée vers 350 apr. J.-C., c.-à-d. époque protomédiévale sans importance versus rôle décisif de l'époque protomédiévale selon WÜEST (ID., 2008 : 377). Attention : Quelques pages après (ID., IBD. : 382) constate autre chose. Nous en traitons plus bas. 
Herman, à quel point l'apport du Moyen Age et l'influence franque étaient importants en ce qui concerne l'origine des parallélismes langagiers entre la langue d'oïl et les parlers padano-rhétoromans, cf. Wüest $(2008: 377)$ : «Interessanter sind für uns jene Entwicklungen, bei denen Nordfrankreich als Innovationszentrum erscheint, das in die Rätoromania und nach Norditalien ausstrahlt. Diese Entwicklungen gehen wohl auf die Merowingerzeit zurück. ${ }^{17}$

Il tient à mettre en relief - dans son résumé voire son interprétation - que les relations historiques entre le domaine d'oïl et celui de la Padania/Rhétoromania remontent à l'antiquité, cf. Wüest (2008:382) :

Dass das Ladinische mehrere sprachliche Erscheinungen kennt, die es mit dem Galloromanischen verbindet, dass sogar Norditalien zusammen mit der Rätoromania in älterer Zeit als ein Teil der Galloromania betrachtet werden können, ist keine Neuigkeit. Wir wollten in diesem Beitrag dagegen aufzeigen, welcher Natur diese Beziehungen waren. Nach unserer Meinung führen sie bis an die Anfänge der römischen Kolonisierung zurück [...]. Sie wären demnach eher älter, als dies Jodl $2004[\ldots]$ annimmt. ${ }^{18}{ }^{99}$

Nous faisons donc face à un travail qui selon son premier paragraphe se propose de mettre en relief le rôle décisif de l'époque mérovingienne (ibd., p. 377 : « interessanter sind für uns ...»= «plus intéressantes sont pour nous ... », voir citation complète plus haut) pour en arriver - à la fin de l'article - à la conclusion que les relations existant entre le domaine d'oïl et l'Italie septentrionale remontaient à l'antiquité, ce qui - d'ailleurs - n'est pas du tout contestable. Toujours est-il que cette dernière remarque de l'article est problématique: elle pourrait donner l'impression aux lecteurs qu'en fin de compte c'est précisément l'époque romaine qui a été décisive. Cette interprétation possible découle de ce que Wüest ne précise pas que ces relations précoces sont restées sans conséquences majeures quant à l'évolution du latin parlé en Gaule. Comme l'a dit Wüest (2008 : 377), et avant lui Cerquiglini ( ${ }^{3} 2007$ : 53s.) et Jodl (2000), (2004) et (2005), c'est l'époque mérovingienne voire franque qui jouera le rôle décisif quelques siècles plus tard. Pour résumer la position de Wüest (2008), nous aimerions l'interpréter de la manière suivante : même s'il y a déjà eu des contacts entre la France et l'Italie septentrionales

${ }^{17}$ «Plus intéressantes sont pour nous les évolutions vis-à-vis desquelles la France septentrionale joue le rôle de centre d'innovations qui rayonne jusqu'en Rhétoromania et en Italie du Nord. Ces évolutions remontent en fait à l'époque mérovingienne.»

${ }_{18}$ « Le fait de considérer que le ladin possède plusieurs traits langagiers qui le relient au galloroman et que l'Italie du Nord et la Rhétoromania ont fait partie de la Galloromania à une époque plus ancienne n'est pas nouveau. Le but de cet article était pourtant de démontrer quel était le caractère de ces relations. D'après nous, elles remontent au début de la colonisation romaine [...]. Elles seraient par conséquent encore plus anciennes que ne le suppose Jodl 2004 [...]. »

${ }^{19}$ Dans ce cas, on ne peut que répéter ce qui a été dit plus haut: que les prédispositions pour des futurs changements phonétiques soient déjà présentes dans le latin rhétique de l'époque impériale ne change rien au fait qu'il reste difficile d'attribuer de si profonds changements à la seule antiquité, sans accorder d'importance à l'époque qui suivit : le Moyen Age (cf. JODL 2008 : 165-174). 
pendant l'antiquité, ce n'est qu'à partir de 500 après J.C. env. que les constellations de contacts langagiers prirent des formes susceptibles d'entraîner des changements linguistiques majeurs.

Mais il faut être prudent - ce n'est qu'une interprétation, le texte lui-même ne nous donne pas explicitement cette information. Quoi qu'il en soit des problèmes d'interprétation de cette argumentation en générale - la mise en relief de l'antiquité comme facteur décisif présentée ici par Wüest nous rappelle l'argument de Hilty et nous incite une autre fois à nous demander pourquoi l'on recourt très souvent à l'antiquité pour individuer les facteurs ayant conduit aux changements langagiers qui nous intéressent.

\section{La théorie des strates et le phénomène du changement linguistique : résumé des implications méthodologiques - mono- versus polycausalité}

A d'autres occasions. cf. surtout Jodl (2008: 167-175) mais aussi Id. (2004 : $107+141-144)$ et Id. (2005: $178+183 \mathrm{~s}$.), nous avons essayé de démontrer que dans de nombreux cas les facteurs extralinguistiques tels que le contact linguistique jouent - au sein d'un ensemble de facteurs internes et externes - le rôle de facteur déclencheur (cf. Jodl 2008: 167-175), donc un rôle à ne pas sous-estimer dans le cadre de l'ensemble de facteurs susmentionné. Nous avons dit plus haut que la théorie des strates se prête particulièrement bien pour expliquer le phénomène du changement langagier: la strate qui nous intéresse (la strate "principale" si on veut) et dont nous suivons l'évolution à travers les siècles est le latin. Exporté en Gaule et ayant été adopté par les Gaulois, ce latin va déjà subir certaines modifications dues à la strate (c'est-à-dire la langue originale des Gaulois) qui exerce une influence sous-jacente "par en dessous" (substrat) qui, en tant que strate secondaire (substrat), va disparaître. La strate qui va survivre est le latin, dans sa variante "celtisée", le "gallolatin" ${ }^{20}$. Les changements langagiers initiés par le contact avec le gaulois se renforceront pendant plusieurs siècles, aussi à travers des processus internes à la langue, jusqu'à l'avènement de l'époque franque qui crée une situation de contact langagier encore plus prononcée, cf. Cerquiglini ( ${ }^{3} 2007$ : 53) ou bien Jodl (2004).

Les Francs apprirent donc un latin qui était devenu du gallolatin. Et ce latin déjà pré-transformé va subir une autre transformation par le contact avec la langue francique. Là aussi, c'est le contact langagier qui déclenche ou renforce des changements langagiers qui, une fois initiés, peuvent aussi suivre, comme on l'a dit, des voies indépendantes de facteurs extralinguistiques. Et cette nouvelle strate secondaire, le superstrat ne va lui non plus pas survivre ; c'est la strate principale, le latin (c'est-à-dire le gallolatin francisé), qui survivra - jusqu'à nos jours. Si cette strate principale avait été privée des contacts langagiers auxquels elle fut exposée,

${ }^{20}$ Cf. en bas, paragraphe 4.1 . 
son évolution aurait pris un cours complètement différent et le français d'aujourd'hui aurait une forme toute différente.

Peut-être faut-il répéter que ce ne sont que les changements majeurs qui nous intéressent, c'est-à-dire les changements qui ont fait naître des traits langagiers distinctifs. Étant donné que ces changements sont particulièrement profonds, les chercheurs ont entretemps du mal à les attribuer à une seule cause et recourent, comme on l'a dit pour Schmitt (1974: 61s., cité plus haut), à des explications polycausales. Dans notre thèse de doctorat, Jodl (2004), voire dans Jodl (2005), nous avons suivi la même voie à propos de l'origine de la PKA francoromane en prenant en considération les facteurs suivants, cf. aussi Jodl (2005: 178) :

a.) l'existence d'un allophone palatal de $/ \mathrm{a} /$ en latin celtique $=$ tendance interne au latin qui a pu plus tard, conjointemente avec certaines influences favorables supplémentaires, favoriser la naissance de la PKA en gallolatin

b.) la particularité phonétique du nexus francique de $-\mathrm{k}(\mathrm{k}) \mathrm{ja}$-, c'est-à-dire une tendance à la palatalisation

c.) le contribut $\mathrm{du} / \mathrm{e}^{1 /}$, son typiquement germanique voire francique qui pouvait favoriser la palatalisation du [á] gallolatin qui, elle, pouvait favoriser la PKA

d.) la tendance à centraliser le /a/ atone en francique

e.) l'effet renforçant de la deuxième palatalisation pan-romane

f.) la force articulatoire de l'accent initial en général (début syllabe)

Pour ce qui en est de la situation particulière à laquelle nous devons faire face en Picardie, en Normandie et dans la «Romania submersa», nous renvoyons à Jodl (2004 : 108-115).

La démarche que nous avons étayée dans notre thèse de doctorat repose sur le principe selon lequel une tendance interne ${ }^{21}$ à la langue dont on examine l'évolution à travers les temps ne saurait suffire à elle seule à engendrer des changements phonétiques majeurs. C'est sous l'effet des influences extérieures qui viennent se greffer sur ces tendances internes que les changements s'imposeront, cf. Jodl (2008 : 165-175). Hilty a appliqué plus ou moins le même modèle pour rectifier l'explication monocausale que Wartburg a donnée au sujet de la diphtongaison spontanée advenue en francoroman, cf. Hilty (1992: surtout 120s.). Vu que dans ce cas-là il s'agit d'un principe universel, on serait en droit de l'appliquer aussi à la naissance du protoromanche.

Après avoir exposé quelques opinions corroborant l'importance de l'époque du début du Moyen Age, nous allons encore une fois nous poser la question cruciale de savoir s'il est admissible de négliger une époque toute entière marquée de surcroît par la naissance de contacts linguistiques très intenses et donc d'un intérêt majeur pour les linguistes. Servons-nous encore une fois des Grisons comme exemple et revenons au travail de Hilty (2008) déjà cité. Le but principal de ce tra-

\footnotetext{
${ }^{21}$ Nous tenons à signaler ici que même une tendance interne à une langue peut en fin de compte résulter d'un phénomène de contact comme la variante palatale de [a] qui existait en gallo-latin.
} 
vail de Hilty a été de démontrer que le protoromanche était déjà consolidé dès l'an 350 après J.C. env. et que les chercheurs tels que Walser ont tort de supposer que les Grisons ont été latinisés au Moyen Age, et non pas dès l'antiquité.

Quels enseignements pouvons-nous tirer de cette vision des choses du point de vue méthodologique ? Comme on a déjà signalé plus haut, la mise en relief de l'antiquité comme époque charnière pour l'apparition des innovations langagières voire des changements linguistiques qui ont conduit à la naissance des langues romanes, comme le proto-romanche dans notre cas précis, implique que les époques qui ont suivi n'ont pas contribué de manière décisive à ce processus. Une telle opinion comporte pourtant le danger d'un retour aux explications monocausales - stratégies qu'on avait déplorées longtemps parce qu'aucune des tentatives entreprises pour expliquer de tels phénomènes de manière monocausale n'a fait l'unanimité dans le monde académique. Mais il y a encore autre chose : pourquoi toujours l'antiquité comme point de départ pour la naissance d'une langue? Le prestige d'une langue dépend-il de l'époque à laquelle remonte son origine?

\section{Problèmes de terminologie - problème de perception ? «gallo- " versus «franco-»}

Si l'on tient donc compte du fait que l'éthno-préfixe « gallo-», combiné avec l'adjectif « roman », a pu s'établir comme terme consacré tandis que son corrélatif de « francoroman » n'a même pas fait son entrée dans les dictionnaires, nous nous voyons obligés d'en déduire - comme nous l'avons fait plus haut pour le protoromanche - que pour ce qui est de la naissance du français et de la valorisation des effets des contacts linguistiques, les linguistes attribuent là aussi plus de poids au substrat et par conséquent à l'époque de l'antiquité.

C'est pour celà qu'on serait en droit de se demander s'il n'est pas dangereux de trop forcer le "gallo-» : ne commet-on pas une double erreur en appliquant le terme scientifique consacré de " galloroman » entre autres au français moderne ?

a.) D'une part parce qu'on ignore alors totalement le facteur crucial, c'est-àdire un superstrat par excellence

b.) D'autre part parce que le terme de «galloroman » pourrait représenter une contradiction en soi : "gallo- » appartient à l'antiquité alors que «-roman » fait référence au Moyen Age $^{22}$

$\mathrm{Si}$ on voulait être parfaitement rigoureux, il faudrait entièrement bannir le terme "galloroman » et le remplacer par " gallolatin». Pour s'épargner une telle mesure et maintenir un terme qui s'est quand même établi au fil des siècles, on pourrait trouver le compromis suivant: Il faudrait probablement distinguer entre deux conceptions distinctes du terme « galloroman ». D'une part, il est utilisé pour regrouper les trois grandes unités linguistiques à substrat celtique, que sont les par-

\footnotetext{
${ }^{22}$ Pour les problèmes du "passage du latin au roman" cf. WRIGHT (1996).
} 
lers d'oïl, les parlers d'oc et le francoprovençal. Dans ce cas, il est légitime de se servir du terme "galloroman », à condition de le faire par rapport à des contextes qui traitent de l'époque antérieure à l'arrivée des francs. Si, d'autre part, on l'emploie comme synonyme pour désigner « le français », il vaut mieux se méfier : comme nous l'avons vu précédemment, c'est cette dernière conception qui peut poser problème.

\section{Conclusions}

Pour trouver des explications convaincantes quant à des changements linguistiques ayant eu lieu dans des époques qui nous empêchent l'accès direct on ne peut pas se passer ni d'un seul facteur potentiel. Il s'est même avéré que ce sont les explications polycausales qui sont les plus prometteuses. Mais force est de constater que dans bien des cas cette règle n'a pas été respectée.

La surestimation du rôle joué par le substrat et par conséquent du rôle de l'antiquité pour expliquer la naissance du français transparait dans le fait que l'ethno-préfixe « gallo- » s'est établi comme terme consacré, ce qui n'est pas le cas pour son corrélatif « franco- ». Mais ce même phénomène concerne aussi d'autres problèmes de glottogénèse comme celui de la naissance du protoromanche.

Pour les deux cas - la naissance du (proto-)français et la naissance du (proto)romanche - il faudrait pourtant faire l'objection suivante : Si on suit les avis selon lesquelles les facteurs en vigueur pendant l'époque de l'antiquité seraient les facteurs décisifs pour faire naitre une nouvelle langue, on risque de retomber dans les argumentations monocausales qui ne se sont pas révélées justes elles non plus. C'est-à-dire qu'en excluant l'époque qui a suivi, autrement dit le Moyen Age, on exclut d'emblée tout un faisceau de facteurs qui pourraient éclairer des problèmes linguistiques.

En d'autres termes : Après des décennies de débats théoriques allant d'un extrême à l'autre, tous à base monocausale, les argumentations monocausales ne sont plus prises au sérieux parce qu'elles - nous l'avons dit plus haut - ne se sont pas révélées justes elles non plus. On peut citer en exemple la théorie sur la naissance du frioulan basée sur une argumentation monocausale désormais dépassée qui en ne prenant en compte que le facteur "variétés du latin" et en négligeant l'apport du Moyen Age, fait remonter cette naissance à la latinité particulière d'Aquilée ${ }^{23}$. Un compromis est impératif : Non seulement les prédispositions de l'antiquité mais en plus les influences médiévales et encore d'autres facteurs ont pu engendrer le résultat qui nous intéresse, cf. Schmitt (1974: 61s.) et Jodl (2008:165-174).

Il faut aussi se demander pourquoi certains chercheurs continuent à recourir de nos jours à des argumentations qu'on croyait dépassées. Quoi qu'il en soit s'agissant de la naissance des langues romanes, l'apport du superstrat, donc de

\footnotetext{
${ }^{23}$ Cf. Gsell (2003: 342s.) qui met en relief que cette hypothèse n'a pas pu se substantialiser et qui nomme d'autres chercheurs sceptiques par rapport à ce sujet.
} 
l'époque médiévale, a été plus important que l'apport du substrat voire de l'époque antique, cf. Kramer (1999: 19, cité plus haut). Il faudrait réfléchir à renforcer l'usage du terme " francoroman » ce qui affirmerait l'importance du superstrat comme facteur décisif.

\section{Références bibliographiques}

ARGENTE, Joan A.: Sprachen im Kontakt, in: Holtus, Günter/Metzeltin, Michael/Schmitt, Christian (eds.), Lexikon der Romanistischen Linguistik, vol. VII: Kontakt, Migration und Kunstsprachen. Kontrastivität, Klassifikation und Typologie, Tübingen 1998, 1-14.

BANNIARD, Michel (1996): "Die Franken zwischen Spätlatein und Altfranzösisch", dans: Die Franken. Die Wegbereiter Europas. Katalog zur Ausstellung im Reiss-Museum Mannheim, Band I, Mainz: von Zabern, 574578.

BLAIKNER-HOHENWART, Gabriele et alii, éds. (2008): Ladinometria. Festschrift für Hans Goebl zum 65. Geburtstag, Salzburg/Bozen/Vich (TN)/San Martin de Tor (BZ): Universität Salzburg - Fachbereich Romanistik/Freie Universität Bozen/Istitut Cultural Ladin "majon di fascegn" (Vich)/Istitut Ladin "Micurà de Rü" (San Martin de Tor), 2 volumes.

CERQUIGLINI;Bernard, La naissance du français, Paris, PUB, ${ }^{3} 2007$ (Que sais-je 2576).

DARDEL de, Robert/WÜEST, Jakob (1993): „Les systèmes casuels du protoroman. Les deux cycles de simplification“, dans: Vox Romanica 52, 25-65.

GSELL, Otto (1996): „Chronologie frühromanischer Lautwandel“, in: Holtus, Günter/Metzeltin, Michael/Schmitt, Christian (eds.): LRL = Lexikon der Romanistischen Linguistik. Vol. II,1: Latein und Romanisch. Historischvergleichende Grammatik der romanischen Sprachen, Tübingen: Niemeyer, 557-584.

- (1997): „Galloromanische Worttypen im ladinisch-padanischen Raum”, dans: Ladinia 21, 135-151.

- (2003): Etymologische und wortgeschichtliche Erforschung und Beschreibung der romanischen Sprachen: Friaulisch, Dolomitenladinisch und Bündnerromanisch“, in: Ernst, Gerhard et alii (éds.): Romanische Sprachgeschichte. Ein internationales Handbuch zur Geschichte der romanischen Sprachen, Berlin/New York: DeGruyter, 339-345. $($ HSK = Handbücher zur Sprach- und Kommunikationswissenschaft 23.1).

HEINEMANN, Sabine (2005, Réc.): „Jodl, Frank (2004): Francia, Langobardia und Ascolis Ladinia. Die Bedeutung außersprachlicher Faktoren im Zusammenhang mit innersprachlichen Entwicklungen in drei Teilgebieten der Romania. Frankfurt/Main: Verlag Peter Lang.“, dans: Vox Romanica 64, 214218. 
HILTY, Gerold (1992): „Aspetti del superstrato germanico del gallo-romanzo“, dans: Quaderni del dipartimento di linguistica e letterature comparate 8, Bergamo: Università degli Studi, 117-128.

- (2008): „Wann wurde Graubünden wirklich romanisiert?“, dans: Gabriele Blaikner-Hohenwart et alii (éds.), vol. 1, 215-228.

- (en voie de publication): „La storia del romancio e la ,questione ladina'“, dans: Actes du XXV Congrès International de Linguistique et Philologie Romanes 2007, Innsbruck.

JODL, Frank (2000): „Churrätien und das Frankenreich in sprachlicher Hinsicht:

Das Zusammenspiel ausser- und innersprachlicher Faktoren und mögliche Folgen für die Herausbildung des Bündnerromanischen" in: Annalas da la Societad retorumantscha 113, 109-142.

- (2004): Francia, Langobardia und Ascolis Ladinia. Die Bedeutung außersprachlicher Faktoren im Zusammenhang mit innersprachlichen Entwicklungen in drei Teilgebieten der Romania. Frankfurt/Main: Verlag Peter Lang.

- (2005): „L'origine della palatalizzazione di [k,g/a] nel romanzo dell'Italia settentrionale, del Ticino, dei Grigioni e della Ladinia dolomitica", dans: Ladinia 29, 155-192.

- (2008): „Alles alte Hüte? Die Konzepte Vulgärlatein, Romanisch und kontaktinduzierter Sprachwandel im Lichte der aktuellen Sprachwandeldiskussion“, dans: Gabriele Blaikner-Hohenwart et alii (éds.), vol. 2, 159-178.

KRAMER, Johannes (1999): „Sind die romanischen Sprachen kreolisiertes Latein?", in: $Z r P h$ 115, 1-19.

KRISTOL, Andres Max (1998): „Die historische Klassifikation der Romania III. Rätoromanisch“, in: LRL VII, Art. 504, 937-948.

LÜDTKE, Helmut (2005): „Von der historischen Grammatik zur Geschichte der Kommunikation", in: Stehl, Thomas (éd.): Unsichtbare Hand und Sprecherwahl. Typologie und Prozesse des Sprachwandels in der Romania. Tübingen: Narr, 49-73.

PFISTER, Max (1995): „Dal latino della Gallia cisalpina agli idiomi romanzi dell'Italia settentrionale", in: Banfi, Emanuele et alii (éds.): Italia settentrionale: Crocevia di idiomi romanzi. Atti del convegno internazionale di studi, Trento 21-23 ottobre 1993, Tübingen: Niemeyer, 189-217.

REISS-MUSEUM (1996): Die Franken - Wegbereiter Europas. Vor 1500 Jahren: König Chlodwig und seine Erben. Katalog-Handbuch zur Ausstellung, 2 voll., Mannheim: Reiss-Museum.

SCHLEMMER, Gerd (1983): Die Rolle des germanischen Superstrats in der Geschichte der romanischen Sprachwissenschaft, Hamburg: Buske (Romanistik in Geschichte und Gegenwart; Bd. 12).

SCHMID, Heinrich (1956): „Über Randgebiete und Sprachgrenzen, III. Über die Palatalisierung von C,G vor A im Romanischen (Zur sprachlichen Stellung Oberitaliens)“", in: Vox Romanica 15/2, 53-80. 
SCHMITT, Christian (1974): „Genèse et typologie des domaines linguistiques de la Galloromania", dans: Travaux de linguistique et de littérature XII, 1, 31-83.

WALSER, Gerold (1944): Studien zur Alpengeschichte in antiker Zeit. Stuttgart: Steiner.

WARTBURG, Walther v. $\left({ }^{5} 1946 /{ }^{12} 1993\right)$ : Evolution et structure de la langue française, Tübingen/Basel : Francke.

WRIGHT, Roger (1996/1991, éd.): „The conceptual distinction between Latin and Romance: invention or evolution?", dans: Wright, Roger (1996/1991, éd.), 103113.

- (1996/1991, éd.): Latin and the Romance Languages in the Early Middle Ages. University Park: The Pennsylvania State University Press 1996 (d'abord publié 1991 chez Routledge).

WÜEST, Jakob (2008): „Ladinisch und Galloromanisch“, dans: Gabriele BlaiknerHohenwart et alii (éds.), vol. 1, 377-384. 